\title{
Reversible reduction of TEMPO radical: One step closer to an all-organic redox flow battery
}

Luke Wylie $^{\mathrm{a}}$, Thomas Blesch ${ }^{\mathrm{a}}$, Rebecca Freeman ${ }^{\mathrm{a}}$, Kan Hatakeyama-Sato ${ }^{\mathrm{b}}$, Kenichi Oyaizu ${ }^{\mathrm{b}}$, Masahiro Yoshizawa-Fujita ${ }^{c}$, Ekaterina I Izgorodina ${ }^{\mathrm{a} *}$

${ }^{a}$ School of Chemistry, Monash University, Wellington Rd, Clayton VIC 3800, Australia

${ }^{\mathrm{b}}$ Department of Applied Chemistry, Waseda University, 3-4-1 Okubo, Shinjuku, Tokyo 169-8555, Japan

${ }^{\mathrm{c}}$ Department of Materials and Life Sciences, Sophia University, 7-1 Kioi-cho, Chiyoda-ku, Tokyo 102-8554, Japan *Katya.Pas@monash.edu

Supplementary Information 3 Pages, 2 Tables, 0 Figures: 
Table S1: Protonation enthalpy and Gibbs free energy (GFE) from TEMPO ${ }^{-}$to TEMPOH in $\mathrm{kJ} \mathrm{mol}^{-1}$

\begin{tabular}{llrr}
\hline Cation & Anion & Enthalpy & GFE \\
\hline $\mathrm{C}_{1} \mathrm{mim}^{+}$ & $\mathrm{N}(\mathrm{CN})_{2}{ }^{-}$ & -166.8 & -171.6 \\
$\mathrm{C}_{1} \mathrm{mim}^{+}$ & $\mathrm{Mes}^{-}$ & 17.0 & 14.7 \\
$\mathrm{C}_{1} \mathrm{mim}^{+}$ & $\mathrm{NTf}_{2}{ }^{-}$ & -32.8 & -32.4 \\
$\mathrm{C}_{1} \mathrm{mim}^{+}$ & $\mathrm{BF}_{4}{ }^{-}$ & 17.8 & 16.6 \\
$\mathrm{C}_{1} \mathrm{mim}^{+}$ & $\mathrm{OTf}^{-}$ & -51.0 & -54.6 \\
$\mathrm{P}_{1,1,1,1}{ }^{+}$ & ${\mathrm{N}(\mathrm{CN})_{2}{ }^{-}}_{\mathrm{Mes}^{-}}$ & -87.5 & -88.6 \\
$\mathrm{P}_{1,1,1,1}{ }^{+}$ & $\mathrm{NTf}_{2}{ }^{-}$ & 7.1 & 12.2 \\
$\mathrm{P}_{1,1,1,1}{ }^{+}$ & $\mathrm{BF}_{4}{ }^{-}$ & 47.6 & 48.0 \\
$\mathrm{P}_{1,1,1,1}{ }^{+}$ & $\mathrm{OTf}^{-}$ & 60.4 & 56.5 \\
$\mathrm{P}_{1,1,1,1}{ }^{+}$ & $\left.\mathrm{N}^{-} \mathrm{CN}\right)_{2}{ }^{-}$ & 30.1 & 23.5 \\
$\mathrm{C}_{1} \mathrm{mpyr}^{+}$ & $\mathrm{Mes}^{-}$ & 31.5 & 38.2 \\
$\mathrm{C}_{1} \mathrm{mpyr}^{+}$ & $\mathrm{NTf}^{-}$ & 154.9 & 155.8 \\
$\mathrm{C}_{1} \mathrm{mpyr}^{+}$ & $\mathrm{BF}_{4}{ }^{-}$ & 63.8 & 62.8 \\
$\mathrm{C}_{1} \mathrm{mpyr}^{+}$ & $\mathrm{OTf}^{-}$ & 36.1 & 37.3 \\
$\mathrm{C}_{1} \mathrm{mpyr}^{+}$ & & 198.6 & 193.7
\end{tabular}


Table S2: Interaction energies between ionic liquid ion pair with $\mathrm{TEMPO}^{-}$and TEMPOH in $\mathrm{kJ} \mathrm{mol}^{-1}$

\begin{tabular}{|c|c|c|c|c|c|}
\hline Nitroxide & Cation & Anion & Electrostatic & Correlation & Total \\
\hline Aminoxy Anion & $\mathrm{C}_{1} \mathrm{mim}^{+}$ & $\mathrm{N}(\mathrm{CN})_{2}^{-}$ & -72.8 & -62.1 & -134.9 \\
\hline Aminoxy Anion & $\mathrm{C}_{1} \mathrm{mim}^{+}$ & $\mathrm{Mes}^{-}$ & -107.8 & -74.2 & -181.9 \\
\hline Aminoxy Anion & $\mathrm{C}_{1} \mathrm{mim}^{+}$ & $\mathrm{NTf}_{2}^{-}$ & -172.4 & -54.0 & -226.5 \\
\hline Aminoxy Anion & $\mathrm{C}_{1} \mathrm{mim}^{+}$ & $\mathrm{BF}_{4}^{-}$ & -125.1 & -57.5 & -182.6 \\
\hline Aminoxy Anion & $\mathrm{C}_{1} \mathrm{mim}^{+}$ & $\mathrm{OTf}^{-}$ & -103.7 & -72.9 & -176.6 \\
\hline Aminoxy Anion & $\mathrm{P}_{1,1,1,1}{ }^{+}$ & $\mathrm{N}(\mathrm{CN})_{2}^{-}$ & -134.2 & -71.4 & -205.6 \\
\hline Aminoxy Anion & $\mathrm{P}_{1,1,1,1^{+}}$ & $\mathrm{Mes}^{-}$ & -68.9 & -64.7 & -133.6 \\
\hline Aminoxy Anion & $\mathrm{P}_{1,1,1,1}{ }^{+}$ & $\mathrm{NTf}_{2}^{-}$ & -149.1 & -74.5 & -223.6 \\
\hline Aminoxy Anion & $\mathrm{P}_{1,1,1,1}{ }^{+}$ & $\mathrm{BF}_{4}^{-}$ & -127.3 & -75.4 & -202.7 \\
\hline Aminoxy Anion & $\mathrm{P}_{1,1,1,1}{ }^{+}$ & OTf $^{-}$ & -134.1 & -77.6 & -211.7 \\
\hline Aminoxy Anion & $\mathrm{C}_{1} \mathrm{mpyr}^{+}$ & $\mathrm{N}(\mathrm{CN})_{2}^{-}$ & -122.6 & -81.7 & -204.2 \\
\hline Aminoxy Anion & $\mathrm{C}_{1} \mathrm{mpyr}^{+}$ & $\mathrm{Mes}^{-}$ & -114.5 & -53.6 & -168.2 \\
\hline Aminoxy Anion & $\mathrm{C}_{1} \mathrm{mpyr}^{+}$ & $\mathrm{NTf}_{2}^{-}$ & -142.7 & -79.7 & -222.3 \\
\hline Aminoxy Anion & $\mathrm{C}_{1} \mathrm{mpyr}^{+}$ & $\mathrm{BF}_{4}^{-}$ & -130.3 & -51.5 & -181.9 \\
\hline Aminoxy Anion & $\mathrm{C}_{1} \mathrm{mpyr}^{+}$ & $\mathrm{OTf}^{-}$ & -144.3 & -48.6 & -193.0 \\
\hline TEMPOH & $\mathrm{C}_{1} \mathrm{mim}^{+}$ & $\mathrm{N}(\mathrm{CN})_{2}^{-}$ & -27.0 & -68.3 & -95.3 \\
\hline TEMPOH & $\mathrm{C}_{1} \mathrm{mim}^{+}$ & $\mathrm{Mes}^{-}$ & -35.1 & -66.8 & -101.9 \\
\hline TEMPOH & $\mathrm{C}_{1} \mathrm{mim}^{+}$ & $\mathrm{NTf}_{2}^{-}$ & -18.3 & -70.4 & -88.7 \\
\hline TEMPOH & $\mathrm{C}_{1} \mathrm{mim}^{+}$ & $\mathrm{BF}_{4}^{-}$ & -37.6 & -57.4 & -95.0 \\
\hline TEMPOH & $\mathrm{C}_{1} \mathrm{mim}^{+}$ & $\mathrm{OTf}^{-}$ & -24.5 & -70.5 & -95.1 \\
\hline TEMPOH & $\mathrm{P}_{1,1,1,1}^{+}$ & $\mathrm{N}(\mathrm{CN})_{2}^{-}$ & -24.1 & -51.1 & -75.2 \\
\hline TEMPOH & $\mathrm{P}_{1,1,1,1}{ }^{+}$ & $\mathrm{Mes}^{-}$ & -42.3 & -56.6 & -99.0 \\
\hline TEMPOH & $\mathrm{P}_{1,1,1,1}{ }^{+}$ & $\mathrm{NTf}_{2}^{-}$ & -18.9 & -69.6 & -88.5 \\
\hline TEMPOH & $\mathrm{P}_{1,1,1,1}{ }^{+}$ & $\mathrm{BF}_{4}^{-}$ & 28.3 & -56.0 & -27.7 \\
\hline TEMPOH & $\mathrm{P}_{1,1,1,1}{ }^{+}$ & $\mathrm{OTf}^{-}$ & -33.5 & -61.4 & -94.9 \\
\hline TEMPOH & $\mathrm{C}_{1} \mathrm{mpyr}^{+}$ & $\mathrm{N}(\mathrm{CN})_{2}^{-}$ & -60.6 & -75.8 & -136.4 \\
\hline TEMPOH & $\mathrm{C}_{1} \mathrm{mpyr}^{+}$ & $\mathrm{Mes}^{-}$ & 23.6 & -52.6 & -29.0 \\
\hline TEMPOH & $\mathrm{C}_{1} \mathrm{mpyr}^{+}$ & $\mathrm{NTf}_{2}^{-}$ & -63.1 & -79.3 & -142.4 \\
\hline TEMPOH & $\mathrm{C}_{1} \mathrm{mpyr}^{+}$ & $\mathrm{BF}_{4}^{-}$ & -83.0 & -50.7 & -133.7 \\
\hline TEMPOH & $\mathrm{C}_{1} \mathrm{mpyr}^{+}$ & $\mathrm{OTf}^{-}$ & 21.0 & -49.1 & -28.1 \\
\hline
\end{tabular}

